

\section{Darle cuerpo a lo no patológico: estigmas de degeneración atribuidos a la locura en Colombia, finales del siglo XIX y principios del XX'}

Give body to the non-pathological: stigmas of degeneration attributed to madness in Colombia, late nineteenth and early twentieth centuries // Dar corpo ao não-patológico: estigmas de degeneração atribuídos à loucura na Colômbia, no final do século XIX e início do século XX

Jairo Gutiérrez Avendaño²

Universidad Católica Luis Amigó, Colombia

jairo.gutierrezav@amigo.edu.co

Revista Corpo-grafías: Estudios críticos de y desde los cuerpos / Volumen 7 - Número 7 / Enero diciembre 2020 / ISSN impreso 2390-0288, ISSN digital 2590-9398 / Bogotá, D.C., Colombia / pp. 238-247

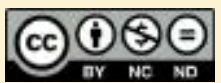

Fecha de recepción: 15 de enero de 2019

Fecha de aceptación: 1 de mayo de 2019

DOI: https://doi.org/ 10.14483/25909398.15521

Cómo citar este artículo: Gutiérrez Avendaño, J. (2020, enero-diciembre). Darle cuerpo a lo no patológico: estigmas de degeneración atribuidos a la locura en Colombia, finales del siglo XIX y principios del XX. Revista Corpo-grafías: Estudios críticos de y desde los cuerpos, 7(7), pp. 238247 / ISSN 2390-0288.

1 Articulo de reflexión Investigación: El presente artículo deriva de la tesis: Locura y Sociedad. Alienismo tardío, psicopatología e higiene mental en la modernidad colombiana, 1870-1968, aprobada en el Doctorado en Ciencias Humanas y Sociales de la Universidad Nacional de Colombia. Inscrita en la Convocatoria de Financiación de Proyectos de Investigación, 2018, Universidad Católica Luis Amigó (Medellín, Colombia).

2 Doctor en Ciencias Humanas y Sociales, Universidad Nacional de Colombia. Posdoctorado en Bioética. Magíster en Educación, Filósofo. Docente investigador del grupo Estudios de Fenómenos Psicosociales, Universidad Católica Luis Amigó (Medellín, Colombia). Integrante de la Red Iberoamericana de Historia de la Psiquiatría y de la Red Colombiana de Historia de la Salud Mental. 


\section{Resumen}

El artículo presenta un seguimiento de los estigmas de degeneración atribuidos a la locura en Colombia, en casos representativos de finales del siglo XIX y principios del XX. El artículo se desarrolla a partir de la consulta de documentos de medicina psiquiátrica en Colombia, así como en estudios significativos sobre el tema.

\section{Palabras clave}

Degeneración; psiquiatría; enfermedad mental

\section{Abstract}

The article presents a follow-up of the stigmas of degeneration attributed to madness in Colombia, in representative cases of the late nineteenth and early twentieth centuries. The article is developed from the consultation of documents of psychiatric medicine in Colombia, as well as in sigficativos studies on the subject.

\section{Keywords}

Degeneration; psychiatry; mental illness

\section{Resumo}

$\mathrm{O}$ artigo apresenta um rastreamento dos estigmas da degeneração atribuídos à loucura na Colômbia, em casos representativos do final do século XIX e início do século XX. O artigo é desenvolvido a partir da consulta de documentos de medicina psiquiátrica na Colômbia, bem como em estudos significativos sobre o assunto.

\section{Palavras-chaves}

Degeneração; psiquiatría; doença mental

\section{Cuerpo ampliado y carga psicopática familiar}

La universal teoría de la degeneración se atribuye al psiquiatra austro-francés Benedict A. Morel en el Traité des dégénérescences physiques, intellectuelles et morales de l'espèce humaine..., publicado en 1857. En la segunda parte de los prolegómenos, se refiere a las "Consideraciones generales sobre los principios que deben guiar la medicina en el tratamiento de las degeneraciones" y establece que "los seres degenerados forman grupos o familias que poseen elementos distintivos en la naturaleza de la causa que los ha hecho invariablemente lo que son en realidad: un desvío mórbido del tipo normal de la humanidad" (Morel, 1857). De las fuentes analizadas en la presente investigación, para el contexto colombiano, emergieron una serie de clasificaciones de la degeneración: a) médico-legal, b) manicomial, c) etiológica, d) diagnóstico-patológica, e) profiláctico-terapéutica. Estas se relacionan con seis tipos de causas que clasificó Morel (1857) y que predominaron en el discurso de la decadencia de la raza en el país, tales como:

1) Por agentes intoxicantes:

a. Alcoholismo (capítulo I), sobre el que trata extensos apartados y sus daños físicos, intelectuales, psíquicos y morales. (pp. 79-140).

b. Del reino vegetal y mineral (capítulo II), en el que incluye el consumo de hachisch, opio y tabaco; intoxicación saturnina (por plomo), fósforo, arsénico, mercurio y ergotina. (pp. 141-203)

c. Por sustancias alimentarias alteradas, (capítulo III), como son: el ergotismo convulsivo y gangrenoso (causado por el ergot o cornezuelo que contamina el centeno y otros cereales), la pelagra (o piel áspera), la cual en la época se consideraba una enfermedad infecciosa (pp. 204-268).

d. Paludismo y su determinismo geográfico (capítulo VII), en el que se refiere principalmente a la patología comparada entre regiones (pp. 612-680).

2) Por el medio social, como las producidas por actividades industriales, la miseria y las profesiones insalubres.

3) Por antecedentes de enfermedad o de un temperamento malsano.

4) Por causas físico-morales. 
5) Por enfermedades congénitas o adquiridas en la infancia.

6) Por influencias hereditarias (Caponi, 2012, pp. 85-86).

Dichos factores etiológicos se explicaban mediante analogías y diferencias entre los tipos de agentes en relación con los climas y la perturbación atmosférica y, a su vez, con determinadas civilizaciones y razas; así como las implicaciones de los mismos en la higiene pública y privada. Otras semejanzas se establecían entre lo fisiológico y lo intelectual, al modo de la teoría de los temperamentos, en la que se atribuyen caracteres psíquicos a órganos del cuerpo afectados.

Posterior a Morel, el médico francés Charles Féré, en su obra La famille névropathique, publicada en 1884, amplió dicha teoría más allá de la locura para hacer de ella un modelo explicativo global de la herencia patológica.

Los estados mórbidos propios de cada una de esas dos ramas, los delirios alucinatorios como las atrofias musculares progresivas, las melancolías como las esclerosis en placas tienen, en efecto, por origen común "una enfermedad primordial y única" del sistema nervioso, trasmitida de forma hereditaria. De ahí su coexistencia, tan frecuentemente subrayada en los estudios clínicos, en el seno de las familias taradas (Féré, 1884 [2004], pp. 310-314).

Es así como, Foucault pregunta en su curso El poder psiquiátrico ¿De qué se trata, en el fondo, cuando se pregunta a un enfermo mental cuáles fueron las enfermedades de su familia y se anota con cuidado si su padre murió apopléjico, si su madre sufría de reuma, si su tío tuvo un hijo idiota, entre otras? (Foucault, 1973-1974 [2007], p. 269). En efecto, puede responderse desde las historias clínicas de los asilos psiquiátricos de Colombia, en las que predominó como primer ítem el de "antecedentes", en el que por lo general se consignaba, "muy recargada de herencia psicopática por los colaterales de sus ascendientes", así como "por consanguinidad", fami- lia con antecedentes: "neuropáticos", "de alcoholismo", "contagio venéreo", "padre loco", "madre loca", entre otros parientes "atacados de perturbación mental".

La familia fue asumida como un "cuerpo ampliado" al que se atribuyó la herencia como marcador biológico, "una especie de sustrato metaorgánico, pero que constituye el verdadero cuerpo de la enfermedad [mental]" Foucault, [2007], p. 311). La higiene física y mental, como solución técnica de la degeneración, hizo énfasis en la preservación de la familia sana para impedir la propagación de la decadencia física, moral y mental en que se encontraba sumida la nación.

Así lo establecía Pablo García Medina en su Tratado elemental de higiene... (1907-1915), en el que reafirmaba la "degeneración por agentes intoxicantes" planteada por Morel, con mayor relevancia el alcoholismo considerado uno de los principales problemas a intervenir, en tanto los hijos de alcohólicos heredaban "una predisposición a las enfermedades nerviosas, como la epilepsia, la degeneración intelectual y el idiotismo [...] heredan tendencia al vicio de esas bebidas, de manera que el alcoholismo se perpetúa y degenera a la familia" (García, 1915).

De este modo, la degeneración y la locura fueron incluidas en el grupo de las patologías sociales (alcoholismo y toxicomanías, sífilis, pobreza, delincuencia, "mala educación", y otras) tanto en el discurso científico de la época, como en el criterio de "antecedentes personales" de las historias clínicas de antiguos psiquiátricos colombianos.

\section{Estigmas y determinismos raciales}

La predisposición hereditaria se identificaba por la evidencia frenopática de las cachets typiques o stigmatas (Morel, 1857, p.37), marcas o signos de degeneración que caracterizan las diferentes familias y grupos de degenerados. Estos indicadores patognómicos son también aceptados en la tesis de Lisandro Reyes: "[...] en lo físico, la degeneración se manifiesta desde el nacimiento por estigmas que se reconocen fácilmente" (1890, [1896], 


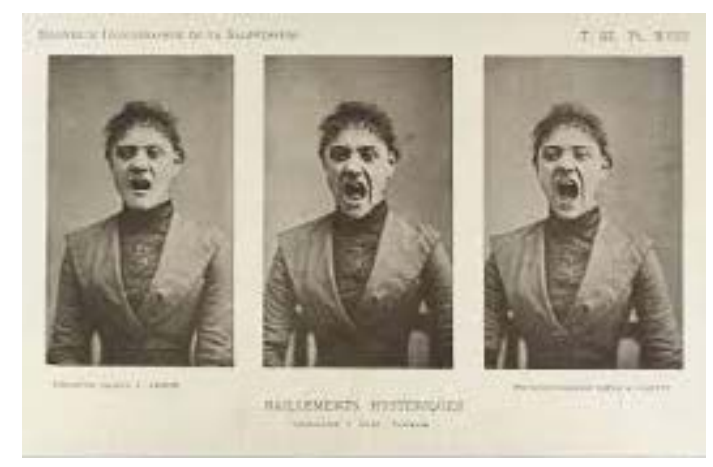

p. 3). A esta forma de diagnóstico, basada en la observación de los cuerpos y en su consecuente clasificación, se recurre también en varias tesis de medicina de la época, que siguieron el primer capítulo del tratado de Alexandre Cullerre (1849-1934), ${ }^{3}$ Les frontières de la folie de (1888), La folie, l'hérédité et les dégénérescenses Intellectuelles, en el que el médico francés recurre a los "Stigmates physiques, intellectuels et moraux de la dégénérescence héréditaire." En la misma vía fueron citadas otras obras de Cullerre: Nerviosisme y névrose (1887) y Traité practique des maladies mentales (1890).

Por otra parte, sobre la neurastenia, Rodríguez recurrió al texto de Jules Dallemagne (1858-1923), ${ }^{4}$ Dégénérés et déséquilibrés (1895). Este autor francés era reconocido por sus observaciones sobre los estigmas anatómicos, biológicos y sociológicos de la criminalidad. En la misma orientación teórica, Rodríguez citó también el tratado La famille névropathique (1884) de Charles Féré (1852-1907). ${ }^{5}$ Féré extendió la teoría de Morel más allá de la alienación mental, para hacer de ella un

3 Médico, director de Asile d'aliénés de la Roche-sur-Yon. Miembro de la Société médico-psychologique.

$4 \quad$ Profesor de medicina legal en Université libre de Bruxelles.

$5 \quad$ Alienista, en 1881 comenzó a trabajar como asistente de Charcot, en el Hospital de la Pitié-Salpêtrière de Paris. Fue nombrado jefe del laboratorio del Hospice de Bicêtre en 1887, donde permaneció el resto de su de carrera.
Imagen 2. La Salpêtrière, "hogar" de locas, prostitutas, mendigas o criminales. Nouvelle iconografie de La Salpetriere. Imágenes de archivo, (1888-1918)

(tomada de: http://www.vagabunda.mx/la-salpetriere-hogar-locas-prostitutas-mendigas-criminales/)

modelo explicativo global de la herencia patológica y de la prevalencia de las enfermedades nerviosas en las familias degeneradas (Féré, 1884 [2004]). Asimismo, en la tesis de Aguirre sobre epilepsia se cita el tratado Dégénérescence et criminalité (1888) del mismo Féré.

Aunque las cifras de degeneración no fueron significativas en estadística, permiten ver que cientos de diagnósticos de degeneración estuvieron asociados con "debilidad mental" (idiocia, imbecilidad, cretinismo, entre otros), alcoholismo, locura moral, epilepsia, demencia precoz, psicosis y manías. No obstante, esta nosología será influyente en la formación teórica de la higiene mental y psiquiatrización de la infancia en adelante. Asimismo, el degeneracionismo, asumió que la nación se encontraba en un estado pueril, analogía del infantilismo al que se atribuían expresiones como retraso físico y mental, raquitismo, discapacidad y primitivismo de la población, mito que será comentado más adelante.

En la historia de la locura era recurrente el reduccionismo al complejo de colonización, que asumía el fenómeno de la locura asociado con el mito del primitivismo, al igual que la degeneración de la raza, como lo plantearon los discursos médicos y políticos de la primera mitad del siglo XX, como se mencionó atrás sobre la inclinación al delirio religioso, así como en el diagnóstico de debilidad mental y será retomado más adelante con respecto a la 
infancia anormal. Frente a esta actitud, en 1913, Putnam atribuía como causa del atraso, entre otros factores ambientales, a la pervivencia de "hábitos de la prehistórica familia indígena, con sus atavismos ancestrales" (pp. 248-259), tal cual lo refirió Jiménez López, sobre la herencia de los grupos étnicos originarios: indígena, europeo, criollo" (1920, p. 27); al igual que Lázaro Uribe Cálad, en 1923, quien, consideraba que:

\begin{abstract}
Los conquistadores de estas comarcas trajeron en sus venas la sangre de Doña Juana la Loca y de aquel gran monarca que sufrió la obsesión de la cronometría [Alfonso X, El Sabio]; aventureros nacidos en las tierras de la brujerías y de los sortilegios; tierra madre del más genial de los sistematizados reivindicadores que registran las crónicas psiquiátricas; soldados tan megalómanos como fanáticos, capaces de las más estupendas hazañas como de actos del más refinado sadismo; conquistadores que mezclaron su sangre ardiente con la del aborigen apático y melancólico, preparado ya para las degeneraciones físicas por sus bárbaros procedimientos para deformar el cráneo (pp. 188-198).
\end{abstract}

En contra de este tipo de postulados, el médico Emilio Robledo, no estuvo de acuerdo con la consideración de la deformación de los cráneos entre los indígenas como signo de degeneración, en tanto que el sistema de Franz J. Gall, no tendría aplicación en estos grupos y que las deformaciones físicas no eran muy frecuentes en los registros antropológicos y médicos del país (1920, p. 4).

La mayoría de conclusiones del discurso de la degeneración recayeron sobre la infancia "semilla del porvenir", la puericultura y la escuela, guiadas por los preceptos de la educación católica, en la que se centralizó la terapéutica contra la degeneración. Con estas últimas soluciones, coincidía Lázaro Uribe Cálad (1923), para el caso de Antioquia y Caldas, quien consideraba la educación como medio de higiene mental para prevenir y remediar la degeneración.
La asimilación de los determinantes y predisponentes hereditarios, influyó en los preceptos impartidos en la Casa de Menores y Escuela de Trabajo de Antioquia, por parte de Tomás Cadavid - primer director- y David Velásquez - médico-, en los que era frecuente la alusión a la anormalidad y la criminalidad infantil como una patología social, debida a factores como la herencia, la miseria, el alcoholismo y chichismo (consumo de bebida fermentada de maíz), la locura, la sífilis, la mala educación, entre otros.

Asimismo, consideraron imprecisa la clasificación de niños "anormales" porque no era causal, por lo tanto, optaron por la evidencia frenopática del estigma "los que tienen algún defecto importante en el cuerpo o en el alma". De hecho, como se refirió, Morel dio un papel relevante a las cachets typiques o stigmatas, que son marcas o signos de degeneración que caracterizan a las diferentes familias y grupos de degenerados (Morel, 1857, p. 37). Como se refirió, este indicador patognómico también fue asimilado en el grupo de las primeras tesis de psiquiatría de la Universidad Nacional de Colombia, así como por Jiménez y Castro (1920, p. 9), al igual que por Vasco en cuanto a la ortopedia mental (1948, p. 59).

\section{Monstrum in fronte, monstrum in animo: es- pectacularización y contagio, emergencia del sentimiento filantrópico}

Un caso de exhibición de la anormalidad en Colombia se encuentra a finales del siglo XIX, en tiempos de la fundación del Asilo de Indigentes y Locos en el antiguo convento de San Diego en 1870, según lo relata Cordovez Moure (abogado, escritor y político), en sus Reminiscencias de 1899, permitían a locos y anormales salir de los asilos a mendigar, "algunos idiotas monstruosos de ambos sexos... medio cubiertos con ruanas... en tal estado de repugnante desaseo que donde quiera que se detenían dejaban la semilla de la plaga que llevaban consigo" (1899 [1997], pp. 909-910). 


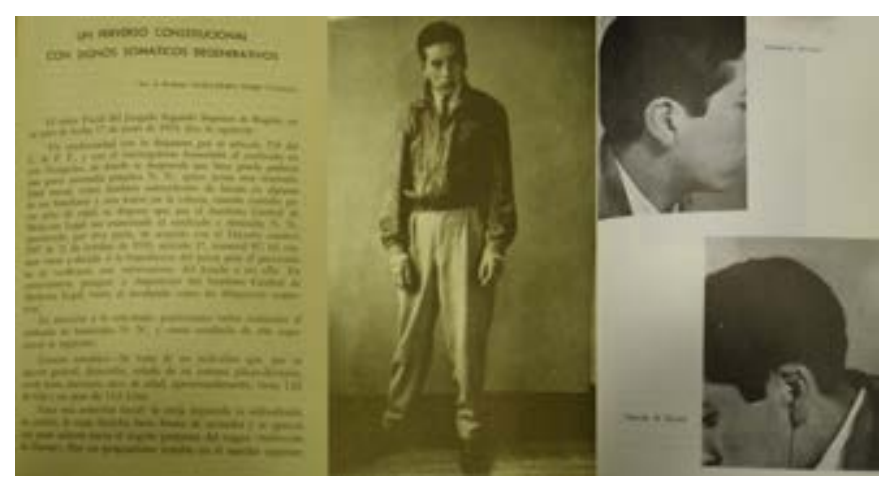

Como personajes de la "corte de los milagros", descritos en Los miserables de Víctor Hugo, se valían de exhibir estigmas y hacer excentricidades para el divertimento de los que pagaban algún centavo por el acto. Así lo expresaba el autor, "iy pensar que había seres racionales para quienes era inocente diversión hacer bailar a esos desgraciados y burlarse de las barbaridades inconscientes que realizaban!" (Cordovez, 1899 [1997] p. 910).

Sobre lo que significaba ser declarado demente en el histórico barrio Guayaquil de Medellín en los años veinte, el texto Moscas de todos los colores... de Jorge Betancur, en un apartado titulado "Enloquecer", muestra el mosaico de personajes que deambulaban por las calles:

Como los volados de casa, como los pordioseros, como los buscadores de trabajo, como los busca fortunas, como las putas, también desquiciados y estúpidos hicieron de las calles de la ciudad su nuevo hogar. La figura de seres con rostros descompuestos, ropas ajadas y sucias, caminar lento y pesado, poco a poco se volvió familiar, en especial en sitios cercanos a la plaza de mercado. A menudo los guardias recogían a dementes de las calles aledañas y los llevaban a las inspecciones de policía. (2000, p. 110)

En el antiguo Asilo de San Isidro en Cali, el primer acto del nuevo director, como el de Pinel en Francia, fue lle-
Imagen 3. Un perverso constitucional con signos somáticos degenerativos (Guillermo Uribe, 1954). Anales Psiquiátrico 8(31)1954).

var a un herrero para cortarles las cadenas a los pacientes que estaban sujetados (Perdomo, 1996, p. 299). La Junta directiva, el mismo año manifestó que era necesario mantener enfermos en calabozos, hacinados de forma infrahumana por falta de drogas, de personal de enfermería y sin tratamiento, "habría que volver a las épocas medievales, a la infame Bedlam, para encontrar escenas similares a las que se presencian a diario en San Isidro." ${ }^{6}$

Esta alusión al Bethlem Royal Hospital que funcionó en Londres desde el siglo XIV y por antonomasia fue vulgarizado para significar que "se armó un Bedlam", un caos, locura, alboroto, y por ser considerado uno de los mayores espectáculos que abría sus puertas para la curiosidad de los que pagaban por ver las "excentricidades" de los internos, incluso por molestarlos para impresionarse con sus reacciones (Fuller y Miller, 2001, pp. 6-22).

De hecho, la categoría de "insensato" concebía no solo a un sujeto interno e invisible, sino a un "monstruo" (del verbo mostrar) que, por su transgresión natural, se

$6 \quad$ Hospital Psiquiátrico Universitarito del Valle, Informe del Presidente de la Junta Directiva del Asilo San Isidro, 1955, dirigido al Director Departamental de Higiene, Fortunato Aljure, octubre 25 de 1955, Archivo Histórico del Hospital Psiquiátrico Universitario del Valle del Cauca (AHPUV). 
exhibía su negación de lo humano, próximo al primitivismo, a la infantilización, brutalidad y animalidad (2009, p. 222). No en vano, un antecedente de este espectáculo fue los "zoos humanos" de las colonias europeas -desde el siglo XIX e incluso posterior a la Declaración Universal de los Derechos Humanos (1948) - en los que enjaularon adultos y niños aborígenes de tribus remotas, por sus fisonomías, indumentarias y artefactos, para ilustración y entretenimiento de los urbanitas.

La Casona de San Isidro no se libró del exhibicionismo que, según el médico Rómulo Mejía, primer director para 1959, "ni siquiera ha faltado aquella [época] en que los enfermos eran mostrados a trueque de unos pequeños honorarios destinados al sostenimiento de los mismos" (Mejía, 1959). Esta disposición la refiere Goffman como un proceso de "mortificación del yo" que deriva de una exhibición contaminadora de tipo físico $(2009$, p. 39) que, más allá del contacto infecto-contagioso, producía una "humillación" del cuerpo o disciplinamiento para el "buen manejo" médico-administrativo de los asilados.

Según el sociólogo, se trataba de un evento anual de transparencia hacia el público, conocido como "Casa abierta", que servía no solo para mostrar una imagen adecuada del cumplimiento de las funciones, sino para presenciar directamente hechos desconocidos $\mathrm{u}$ ocultos del sometimiento al tratamiento, que obligaba a exponer la intimidad y dignidad del enfermo (Goffman, 2009, pp. 35-36). No obstante, Goffman destaca como atributo benéfico, que la apertura institucional a las visitas individuales, la casa abierta y las inspecciones, dieron paso a la integración e interacción de una comunidad que permanecía dividida por los muros de la racionalidad normalizadora (p. 107).

La mentalidad sobre el contagio de la locura se propagó desde la época del "gran miedo", en el siglo XVIII francés que, según Foucault, concibió el asilo como espacio de contención y, al mismo tiempo, "como espectáculo que no amenace a los espectadores, que reúna todas las posibilidades del ejemplo y ninguno de los riesgos del contagio" (2009, p. 33). Esta generalización preventiva marcó el ingreso del médico en el espacio de la internación, no como perito encargado de separar el mal y la enfermedad, crimen y locura, sino más bien como guardián para contener la amenaza y el desvío que acechaba a la sociedad. En efecto, se produjo una sensibilidad de rechazo a la "peste invisible", a "un gran y progresivo mal" (Fuller y Miller, 2001, pp. 1-6), tanto en Inglaterra como en Irlanda, Canadá y Estados Unidos.

\section{Conclusiones}

En el Manicomio Departamental de Antioquia, entre 1903 y 1930, se encuentran seis casos que registraron el dato de "estigma de degeneración", como el de un joven de 18 años, en 1920, con psicosis constitucional, "paladar ojival y asimetría facial", sin antecedentes hereditarios; otro joven de 17 años, en 1921, sufría de psicopatía tóxica, con estigmas y antecedentes de familia psicopática; asimismo, un joven de 17 años, en 1922, era un débil mental con estigmas de degeneración, sin antecedentes hereditarios.

Otras marcas son las de un hombre de 37 años, en 1922, que sufría de confusión mental, visible "asimetría facial y oreja pequeña", sin antecedentes hereditarios; una mujer de 16 años, en 1922, padecía degeneración física y psíquica, tenía "mano simiana, pie grande, irregularidad en la disposición de las orejas, microcefalia", sin antecedentes hereditarios; por último, un hombre de 23 años, en 1922, con manía degenerativa alcohólica, estigmas y familia alcohólica. Como se observa, estas marcas físicas no necesariamente estaban asociadas a un diagnóstico de "degeneración" ni atribuidos a algún tipo de antecedente hereditario.

Según Abel Martínez, estos supuestos signos fueron revaluados como "simples variaciones sin significación patológica y menos patognómica, es decir, dejaron de ser sín- 


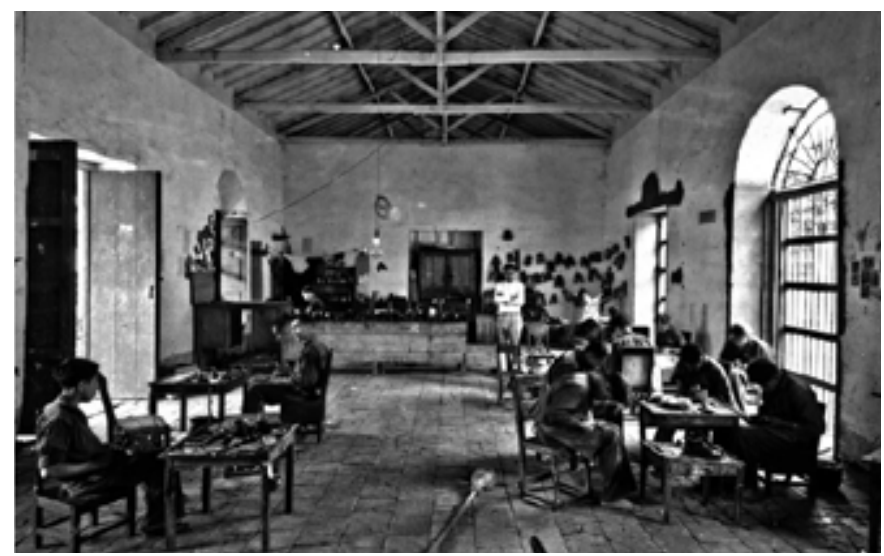

Imagen 4. Casa de menores y escuela de trabajo (fundamento reformatorio y enseñanza de artes y oficios, como la zapateria, para la formación de jóvenes productivos a la sociedad y el autosostenimiento del establecimiento. Archivo fotográfico (Biblioteca Pública Piloto de Medellín). Fotografía: Francisco Mejía.

tomas clínicos, positivas evidencias de degeneración. Las evidencias tampoco demostraron que existiera una degeneración progresiva que llevara a la esterilidad a familias y pueblos como sostenía Morel" (Martínez, 2015, p. 390). Sin embargo, la carga psicopática de la familia siguió fijándose en imaginarios y representaciones sociales que asumieron que esta es productora tanto de salud como de enfermedad, en la que influiría la "genética", "biocodificación", "inconsciente", o como quiera que se trate de un linaje de sangre o designio de los antepasados.

Se evidencia incompatibilidad entre el discurso y la práctica, en tanto la teoría de la degeneración no influyó de manera considerable en la clasificación de los alienados y en sus diagnósticos en los manicomios de Colombia. Esto se constata en el escaso número de diagnósticos de "degeneración". Cuando los hay, estos no se relacionaron con la atribución de las enfermedades mentales a los "antecedentes familiares", criterio que consignaba en las fichas: "familia psicopática", "degenerada" o "alcohólica". Tampoco se relacionaron con los "estigmas frenopáticos" de degeneración que aparecen señalados en unos pocos casos. Asimismo, tampoco es suficientemente significativa la relación de la degeneración con el pronóstico de "incurable".

Estos cuatro registros, diagnóstico, antecedentes, estigmas y pronóstico, tuvieron mayor frecuencia en las primeras décadas de ingresos (1903-1920), veinte años antes del debate sobre la degeneración de la raza en Colombia que tuvo lugar en la década de 1920 y eso que era una teoría vigente en Occidente durante la segunda parte del siglo XIX. Por el contrario, ya en su época de auge empezaron a ser poco visibles en los registros de los asilos del país.

Por otra parte, las evidencias del influjo de la teoría de la degeneración en el surgimiento de la psiquiatría, en particular en el modelo alienista, muestran que en los primeros discursos médicos colombianos dedicados a la psiquiatría se retomaron los predisponentes hereditarios y los factores determinantes del medio en la construcción de una ecléctica etiología de la locura. De lo anterior da cuenta el corpus de 14 tesis sobre psicopatología sustentadas en la Facultad de Medicina de la Universidad Nacional de Colombia, entre 1898 y 1912, las cuales se caracterizaron por el estudio de las causas de la locura y por el principal enfoque de las primeras cátedras de enfermedades mentales en Antioquia (1914) y Cundinamarca (1916).

El declive de la degeneración también se evidencia en lo hallado décadas más tarde en el Estudio Piloto Internacional sobre Esquizofrenia (León y León, 1996, pp. 1235), realizado por la OMS a partir de 1968, el cual contó con una muestra de 100 pacientes del Hospital Psiquiátrico San Isidro del Valle del Cauca, observados durante diez años. El estudio logró desmitificar la idea de que esta era una enfermedad degenerativa y, por el contrario, demostró que un porcentaje significativo de pacientes presentaba mejoría y era socialmente funcional. 


\section{Referencias}

Betancur, J. (2000). Moscas de todos los colores. Historia del barrio Guayaquil de Medellín 1894-1934. Bogotá: Ministerio de Cultura de Colombia.

Caponi, S. (2012). Loucos e degenerados. Uma genealogía da psiquitria ampliada. Manguinhos: Fiocruz.

Cordovez M., José, M. (1899 [1997]). Reminiscencias de Santafé y Bogotá. (Edit.) Gerardo Rivas Moreno. Bogotá: Fundación para la Investigación y la Cultura (FICA).

Féré, Ch. (1884 (2004]). "La famille névropathique. Paris, Delahaye et Lecrosnier". En Pinell, Patrice. Degeneración. En Lécourt, Dominique. (Dir.). Dictionnaire de la pensée médicale. Paris: PUF.

Foucault M. (1973-74 [2007]). El poder psiquiátrico. Curso en el CoIlège de France (1973-1974). Buenos Aires: Fondo de Cultura Económica.

Foucault M. (2009). Historia de la locura en la época clásica.T.1, México: Fondo de cultura Económica.

Fuller, E. y Miller, J. (2001). The invisible plage: the rise of mental illness from 1750 to the present. New Jersey: Rutgers University Press.

García, P. (1915). Tratado elemental de higiene y nociones de fisiología para la enseñanza de estas materias en escuelas y colegios de Colombia. Bogotá: Arboleda y Valencia.

Goffman, E. (2009). Internados. Ensayos sobre la situación social de los enfermos mentales. Buenos Aires: Amorrortu.

Hospital Mental de Antioquia. Archivo pasivo de historias clínicas. Laboratorio de Fuentes Históricas. Medellín: Universidad Nacional de Colombia.

Hospital Psiquiátrico Universitarito del Valle. (1955). Informe del Presidente de la Junta Directiva del Asilo San Isidro. Archivo Histórico del Hospital Psiquiátrico Universitario del Valle del Cauca.

Jiménez, M. (1920). "Nuestras razas decaen. Algunos signos de degeneración colectiva en Colombia y en los países similares". Memoria presentada en el Tercer Congreso Médico Colombiano. (Cartagena, enero de 1918). Bogotá: Imprenta y Litografía de Juan Casis.
León, C., y León, A. (1996). Curso clínico y evolución de la esquizofrenia en Cali. Un estudio de seguimiento de 26 años. Salud Mental, (19), pp. 12-35.

Martínez, A. (2015). “La mayor controversia científica de la intelectualidad colombiana". Miguel Jiménez López y la Degeneración de la Raza (1913-1935). Tesis de Doctorado en Historia. Universidad Pedagógica y Tecnológica de Colombia - UPTC.

Mejía, R. (1959). "La asistencia psiquiátrica en el Valle del Cauca". VI Congreso Médico Nacional (Bogotá, julio 1959). Archivo Hospital Psiquiátrico Universitario del Valle del Cauca.

Morel, B. (1857). Traité des dégénérescences physiques, intellectuelles et morales de l'espèce humaine. Paris: J.

B. Bailliére, Académie Impériale de Médecine, Vol. 1. Disponible en: Bibliothèque Nationele de France, Gallica: http://gallica.bnf.fr/ ark:/12148/bpt6k850762.r=B\%20A\%20Morel

Perdomo, R. (1996). Premio Vida y Obra al servicio de la Psiquiatría otorgado a Carlos A. León. Revista Colombia de Psiquiatría, XXV(4), p. 299.

Putnam, C. (1913). Informe presentado a la Academia Nacional de Medicina. Revista Médica de Bogotá, año XXXI (septiembre de 1913), pp. 248-259.

Reyes, L. (1896). "Contribution à l' étude de l' etat mental chez les enfants dégénérés. Paris: Ollier-Henry, 1890". En: Rodríguez, José. Contribución al estudio de las degeneraciones de evolución. Idiotez. Tesis Facultad de Medicina, Universidad Nacional. Bogotá: Imprenta de Medardo Rivas.

Robledo, E. ¿(1920). ¿Existe una degeneración colectiva en Colombia? Medellín: Tipografía Industrial.

Uribe, L. (noviembre, 1923). Principales factores etiológicos de la locura en los departamentos de Antioquia y Caldas. Revista clínica: órgano de la Sociedad clínica del Hospital de Medellín, 3(25-28), pp. 188-198. 